\title{
Meta
}

Journal des traducteurs

Translators' Journal

\section{KoustAS, Jane (2008) : Les Belles Étrangères : Canadians in Paris, Ottawa, The University of Ottawa Press}

\section{Louis Jolicoeur}

Volume 54, numéro 3, septembre 2009

URI : https://id.erudit.org/iderudit/038322ar

DOI : https://doi.org/10.7202/038322ar

Aller au sommaire du numéro

Éditeur(s)

Les Presses de l'Université de Montréal

ISSN

0026-0452 (imprimé)

1492-1421 (numérique)

Découvrir la revue

Citer ce compte rendu

Jolicoeur, L. (2009). Compte rendu de [Koustas, Jane (2008) : Les Belles Étrangères : Canadians in Paris, Ottawa, The University of Ottawa Press]. Meta, 54(3), 635-637. https://doi.org/10.7202/038322ar

Ce document est protégé par la loi sur le droit d'auteur. L'utilisation des services d’Érudit (y compris la reproduction) est assujettie à sa politique d'utilisation que vous pouvez consulter en ligne.

https://apropos.erudit.org/fr/usagers/politique-dutilisation/
Cet article est diffusé et préservé par Érudit.

Érudit est un consortium interuniversitaire sans but lucratif composé de l’Université de Montréal, l’Université Laval et l’Université du Québec à Montréal. Il a pour mission la promotion et la valorisation de la recherche. https://www.erudit.org/fr/ 
Ce recueil ne manquera pas d'intéresser ceux qui travaillent sur les différents aspects de la collecte et l'analyse des collocations dans les textes spécialisés, en particulier en français, sans oublier les éventuelles extensions plurilingues.

JoHn Humbley

Université Paris 7 - Denis-Diderot, Paris, France

\section{NOTES}

1. Cette tranche du lexique fait l'objet d'un numéro spécial de la Revue française de linguistique appliquée, 12(2), Lexique et écrits scientifiques, 2007, qu'Agnès Tutin a coordonné.

2. La thèse de Tarp Leksikografi i gronselandet mellem viden og ikke-viden. Generel leksikografisk teori med sarlig henblik på lørnerleksikografi, citée par Leroyer, est désormais disponible en anglais: Lexicography in the Borderland Between Knowledge and Non-knowledge: General Lexicographical Theory with Particular Focus on Learner's Lexicography, Niemeyer, coll. «Lexicographica Series Maior», 2008.

3. Corbin, Pierre (2005): Des occurrences discursives aux contextualisations dictionnairiques. Éléments d'une recherche en cours sur l'expression en français d'expériences du football. In: Michaela Heinz, dir., L'exemple lexicographique dans les dictionnaires français contemporains. Actes des Premières Journées allemandes des dictionnaires (Klingenberg-am-Main, 25-27 juin 2004), Lexicographica Series Maior. Vol 128. Tübingen: Max Niemeyer Verlag, 125-156.

Koustas, Jane (2008): Les Belles Étrangères: Canadians in Paris, Ottawa, The University of Ottawa Press.

C'est sans doute le lot des petites nations de se regarder toujours dans le miroir de l'Autre. Le Québec n'est certes pas en reste à cet égard, le miroir parisien battant tout particulièrement la mesure de ses nombreuses angoisses identitaires - la dernière et non la moindre étant sans doute celle qu'aura engendrée le Sommet de la Francophonie tenu à Québec en octobre 2008, où l'on se rappellera à quel point chaque propos du président Sarkozy a été passé à la loupe par des journalistes en mal de crise nationale.

La France est moins connue comme miroir de l'autre solitude canadienne. Or, on oublie qu'elle l'est également, à divers égards, et dans un cadre souvent bien différent de celui où se déploie le jeu ineffable que le Québec entretient avec la mère patrie. C'est ce regard que vient explorer de façon fort intéressante Jane Koustas dans son ouvrage Les Belles Étrangères: Canadians in Paris.

Si c'est d'abord de traduction qu'il s'agit dans ce livre, il ne fait pas de doute qu'il y est aussi question, par le fait même, du regard que les Fran- çais portent de façon plus générale sur le Canada anglais. Du moins, il serait bien limitatif de ne voir ici qu'une revue de la situation de la traduction de la littérature canadienne en France.

Quelques esprits sournois affirment de temps à autre que la littérature québécoise, pour percer en France à sa juste valeur, devrait sans doute tenter de suivre les traces de la littérature du Canada anglais. Or, pour ce faire, il faudrait qu'elle soit une littérature... traduite! S'il est certes attristant de songer que le fait d'écrire en français éloigne peut-être les auteurs québécois de leurs cousins français, voilà tout de même qui donne de belles lettres de noblesse à la traduction! Mais laissons là cette grave question, qui nous entraînerait sur un terrain fort glissant. Tout en observant cependant que la littérature du Canada anglais a bel et bien damé le pion à la littérature québécoise ces dernières années en France, malgré ou grâce au fait qu'il s'agit d'une littérature traduite.

Jane Koustas aborde en filigrane cette question, mais ne s'aventure guère sur les explications possibles de ce fait à priori étrange - cela étant toutefois compréhensible vu la complexité et la sensibilité de cette question. Tel n'est d'ailleurs pas son but premier, qui est d'explorer le contexte de réception de la littérature du Canada anglais en France, ainsi que la place et la nature de la traduction dans le processus de diffusion de cette littérature auprès des lecteurs français. L'objectif de l'auteure est ainsi d'analyser de façon générale l'attitude qu'ont envers la littérature canadienne en traduction les lecteurs français, et plus spécifiquement les traducteurs, les critiques et les éditeurs, en tant que membres d'une même communauté interprétative, qui partagent un même horizon d'attente.

L’ouvrage est divisé en deux parties. La première partie offre une réflexion théorique sur ces questions d'horizon d'attente, de communauté interprétative, ou de lecteur implicite (autant de synonymes en fait pour le contexte de réception), ainsi que sur les concepts de vernacularisation (domestication) et "d'étrangéisation» (foreignization) (termes qui ne manquent pas à leur tour de synonymes). La deuxième partie est consacrée à la situation d'un certain nombre d'écrivains canadiens en France: Mavis Gallant, Nancy Huston, Robertson Davies, Carol Shields, Margaret Atwood, Michael Ondaatje, Ann-Marie MacDonald et Alistair Macleod. L'analyse est intéressante, surtout, m’a-t-il semblé, en ce qui concerne Nancy Huston (dont les déboires avec le prix du Gouverneur général ne pouvaient certes être passés sous silence) et Michael Ondaatje, bien que cela demeure souvent anecdotique. En fait, des conclusions plus percutantes auraient sans doute pu être tirées des cas étudiés si, au-delà des différences en somme accessoires entre traduction québécoise et traduction hexagonale, 
l'auteure avait exploré davantage les liens entre horizon d'attente et manière de traduire, ou entre traduction au sens large et diffusion culturelle.

La sélection des auteurs canadiens étudiés dans l'ouvrage correspond en partie à celle qu'avait faite le festival Les Belles Étrangères en 1996, lorsque le Canada en a été l'invité d'honneur. Ce festival réunit chaque année, en France, une dizaine d'écrivains d'un pays non francophone pour le présenter, en traduction française, au lectorat français. Parmi les auteurs canadiens ayant participé à ce festival en 1996, notons qu'il y avait aussi Neil Bissoondath, que Jane Koustas n'a cependant pas inséré dans sa liste. Dommage d'ailleurs, car ses deux derniers titres ayant été traduits au Québec et distribués en France dans leur traduction québécoise, cela aurait apporté une note quelque peu différente à son analyse, qui insiste fortement sur les difficultés des traducteurs français à bien rendre compte de la réalité canadienne, ainsi que sur la faible propension des éditeurs français à utiliser des traducteurs québécois - deux affirmations qui gagneraient par ailleurs à être quelque peu nuancées.

Koustas ne mentionne que très tard dans son ouvrage qu'il y a tout de même un certain nombre de traductions d'auteurs canadiens réalisées au Québec qui sont distribuées en France, et elle n'analyse guère la chose, ce qui est également dommage. D'autre part, elle insiste beaucoup sur les supposées maladresses des traducteurs français, indiquant qu'on ne devrait jamais accepter que les personnages de Carol Shields ou d'Ann-Marie MacDonald parlent comme s'ils se trouvaient dans le métro de Paris. Or, on peut se demander comment ils devraient alors parler... S'il ne fait pas de doute que bien des traductions françaises pèchent par un excès de confiance et un manque de connaissance de la réalité québécoise (inutile de revenir par exemple sur les traductions françaises de Mordecai Richler), il ne faut pas pour autant s'étonner que les traducteurs s'ajustent à leur lectorat. Le problème, en somme, me semble d'une autre nature. Il me semblerait plus intéressant de chercher en traduction une langue familière qui puisse traverser l'Atlantique sans trop de heurts. Exercice auquel les traducteurs québécois sont par ailleurs bien aguerris. Mais il faut également reconnaître le travail déjà fait en ce sens en France même, plutôt que déplorer à l'excès une situation par ailleurs en constance évolution. En outre, il faut chercher les moyens de donner une plus grande place aux traducteurs québécois de littérature canadienne, sans pour autant s'en prendre aux traducteurs français et à leur tendance, en somme légitime, d'ajuster leurs traductions à l'horizon d'attente de leur lectorat, pour reprendre la terminologie utilisée à répétition par l'auteure.
La France n'est par ailleurs nullement différente des autres pays européens dans ce supposé ethnocentrisme. La tendance à la vernacularisation répétée tout au long du livre de Koustas n'est pas l'apanage de la France et ne me semble d'ailleurs pas particulièrement prononcée dans la France d'aujourd'hui, si on compare les traductions françaises aux traductions italiennes ou espagnoles, par exemple. Sans compter que deux des maisons d'éditions européennes les plus sensibles en matière de traduction sont françaises: Actes Sud et Christian Bourgois. Deux maisons qui non seulement apportent un soin particulier à leurs traductions, mais ont l'audace de traduire bon nombre d'auteurs simplement par souci de faire connaître une «autre littérature», sans toujours penser au premier chef aux ventes escomptées. Il est également étonnant de lire que les traductions en France ne se font pratiquement que par l'intermédiaire d'agents et dans le cadre des foires commerciales comme celle de Frankfort. Si ces foires ont certes leur importance et si les agents font peu à peu leur apparition en France, dans la foulée de ce que l'on observe depuis longtemps dans les milieux du livre anglosaxon ou hispanique, la vieille tradition française du travail solitaire de traducteurs chevronnés et réputés auprès des éditeurs, qui traduisent pour leur plaisir pour ensuite trouver un éditeur de leur choix, n'est pas morte, loin de là. Il s'agit par ailleurs de traducteurs qui ne sont en général nullement enclins à soumettre leurs œuvres à la vernacularisation. Je pense ici en particulier aux grands traducteurs de littérature latino-américaine que sont André Gabastou, Claude Couffon, Annie Morvan et tant d'autres. Il y en a tout autant du côté des traducteurs de littérature anglo-saxonne.

La bibliographie est très complète et constitue un excellent outil de référence. Il est fait état de 640 ouvrages littéraires canadiens traduits en France, sans que soit précisé toutefois qu'un nombre non négligeable de ces traductions ont justement été signées par des Québécois. Il ne s’agit pas ici de se fermer les yeux sur le déséquilibre évident mis en lumière par Koustas, mais bien de reconnaître que s'il y a certes du travail à faire, mieux vaut s'y consacrer en cherchant des voies de solution et en nous inspirant du travail déjà fait. Or, Koustas ne mentionne les traductions québécoises qu'au compte-gouttes, et relève que leur nombre va en augmentant seulement à la toute dernière page de son ouvrage, citant au passage quelques «Quebec translators like Robert Paquin, Robert Lalonde, Lori Saint-Martin, or Marie José Thériault», en précisant que «this very recent development hints at a salutary breakdown of the Quebec/continental French distinction» (p. 124).

Il aurait été intéressant de dresser aussi une bibliographie des romans canadiens traduits au 
Québec, et de mentionner dans la bibliographie proposée dans le livre tous les cas où les traductions, bien qu'éditées en France, ont été réalisées par un traducteur québécois. Cela aurait mis en perspective la thèse défendue un peu radicalement par l'auteure. Je relève dans la bibliographie, outre les noms de Robert Paquin, Robert Lalonde, Lori Saint-Martin et Marie José Thériault mentionnés plus haut, ceux de bon nombre d'autres traducteurs québécois - Hélène Rioux, Jude Des Chênes, Élisabeth Vonarburg, Ivan Steenhout, Jacques Fontaine, Paul Gagné, Charlotte Melançon, Nicole Martel, Émile Martel -, dont rien n'indique qu'ils sont québécois. Et je ne mentionne pas toutes les autres traductions québécoises absentes de la bibliographie, mais dont on sait qu'elles sont également disponibles en France, par exemple les traductions de Mavis Gallant et de Jane Urquhart réalisées par Nicole Côté et publiées par l'Instant même: elles ne sont certes pas en vente dans toutes les librairies de l'Hexagone, mais elles existent tout de même dans le paysage français et peuvent être achetées sans problème à Paris!

$\mathrm{Au}$ lieu de souffler sur les braises d'un supposé «danger of reinforcing stereotypical cultural images, of catering to the horizon of expectations of a foreign target audience, of alienating Canadian and Quebec francophones in order to meet the needs of a continental readership » (p. 124), je crois qu'il eût été préférable de reconnaître le travail des traducteurs et des éditeurs français dans la diffusion de la littérature canadienne en France, de relever davantage la présence tout de même non négligeable des traducteurs québécois dans l'équation, et d'explorer les voies qui pourraient permettre à l'avenir d'accentuer les collaborations d'un côté et de l'autre de l'Atlantique.

Cela étant dit, il s'agit ici d'une recherche importante et novatrice, que l'on souhaiterait étendre à d'autres pays et à d'autres sphères linguistiques. Même si, pour ce qui est de la France seulement, il resterait encore beaucoup à dire.

LOUIS JOLICOEUR Université Laval, Québec, Canada

Wecksteen, Corinne et El Kaladi, Ahmed (2007): La traductologie dans tous ses états. Mélanges en l'honneur de Michel Ballard, Arras, Artois Presses Université, coll. «Traductologie».

La retraite se dit en espagnol jubilación. Et quoi de plus réjouissant comme cadeau de départ que de voir célébrer le travail de toute une vie par des collègues et amis venant des quatre coins du monde? Dix-huit collègues ont donc rassemblé les réflexions que leur avait inspirées l'œuvre du professeur Michel
Ballard dans ce livre traitant des aspects théoriques et pratiques de la traductologie.

Un livre sérieux qui ne se prend pas au sérieux, comme en témoigne le titre de certains articles: «Traduire les textes nobles, traduire les textes ignobles: une seule ou deux méthodes?», «Eau de rose, eau de vinaigre, itinéraire de "vrais amis", ou une écriture à quatre mains. »

Un livre illustrant le rôle de pont que joue la traduction non seulement entre différentes cultures et civilisations, mais également entre diverses époques au sein d'une même culture, ou encore entre différents médias comme le livre et le cinéma. Un livre qui souligne du même souffle que si les ponts rapprochent les hommes, ils rendent plus évidents ce qui les sépare et les contraintes qui en découlent.

Faute de place, il serait impossible de résumer, même en quelques paragraphes, chacun de ces articles. Nous les avons donc rassemblés sous quelques titres pour donner une vue d'ensemble des grands thèmes qui y sont traités.

\section{Traduire la littérature ou les sciences?}

Claude Bocquet et Françoise Wuilmart s'intéressent tous deux à la traduction littéraire, le premier pour se demander s'il existe vraiment une différence fondamentale entre traduction littéraire ou «noble» et traduction technique ou «ignoble», la deuxième pour considérer la traduction littéraire comme source d'enrichissement pour la langue d'accueil.

Il devient de plus en plus évident qu'il ne peut se faire de traduction technique, que ce soit dans le domaine juridique, économique ou autre, sans une bonne connaissance des cultures dans lesquelles ces domaines ont pris naissance et ont évolué. La traduction littéraire, si elle fait, plus que la première, appel à la créativité du traducteur, exige une tout aussi bonne connaissance des cultures d'origine et de destination. Parler de «traduction créative» en l'opposant à «traduction mécanique» constitue donc un faux problème: l'une et l'autre expriment le même souci de rendre compréhensible, au lecteur enraciné dans sa propre culture, la pensée d'un auteur ayant vécu ou vivant dans un autre système culturel.

Comprendre les cultures, c'est donc comprendre ce qui se cache derrière les mots. Et Françoise Wuilmart de le démontrer par le mot français "pain» et son alter ego allemand «brot» qui, pour être une traduction fidèle l'un de l'autre, ne correspondent physiquement ni à la même réalité, ni mentalement au même contexte (beurre et café noir pour l'un; charcuteries et bière pour l'autre). Comprendre ces différences sans chercher nécessairement à les occulter en les acclimatant à sa propre culture, c'est aussi comprendre l'Autre; 